\title{
Anatomic anomaly in renal blunt trauma
}

\author{
Ching-Fang Wu MD, Chun-Chieh Huang MD, Chin-Chi Kuo MD, Che-Hsiung Wu MD, Tze-Wah Kao MD
}

Previously published at www.cmaj.ca

$\mathrm{A}$ nine-year-old girl with cerebral palsy presented with pain in her left flank and gross hematuria that occurred 24 hours after a fall against a chest of drawers. She was alert, and her temperature was $39.2^{\circ} \mathrm{C}$, heart rate 155 beats/min and blood pressure 95/62 mm Hg. Her conjunctivae were pale, and there was tenderness to percussion over the left costovertebral angle. No bruise or wound was seen. Complete blood count showed mild leukocytosis and a hemoglobin level of 90 (normal 120-140) g/L. Other laboratory data, including coagulation profile and renal function, were normal. Blunt injury of the left kidney was diagnosed. Volume expansion with normal saline and two units of packed red blood cells was started immediately.

A contrast-enhanced computed tomography (CT) scan showed a deep, full-thickness parenchymal fracture extending to the pelvis of the patient's left kidney, with minimal urine extravasation (Figure 1). The renal injury was compatible with a grade 4 injury, as defined by the Renal Injury Scale of the American Association of Surgeons in Trauma. ${ }^{1}$ However, the disparity between the deep laceration into the pelvis and minimal urine extravasation implied pre-existing duplicated collecting systems, which were confirmed by CT scanning with three-dimensional reconstruction. Two days after complete bed rest and supportive treatment, the gross hematuria subsided, and the flank pain disappeared. After 18 days in hospital, the girl was discharged home. She will require longterm follow-up, because she is at increased risk of posttraumatic renal hypertension.

Our case supports conservative management of high-grade renal blunt injury (grades 3 to 5) in children. Several reports have found that surgery is required only when a child is hemodynamically unstable or has major associated injuries, or when one kidney is not visible on a contrast-enhanced CT

From the Division of Nephrology, Department of Internal Medicine (ChingFang Wu, Kuo, Kao) and the Department of Medical Imaging (Huang), National Taiwan University Hospital and National Taiwan University College of Medicine, Taipei, Taiwan; and the Division of Nephrology, Department of Internal Medicine (Che-Hsiung Wu), Buddhist Tzu Chi General Hospital, Taipei Branch, Taipei, Taiwan

CMAJ 2010. DOI:10.1503/cmaj.090404

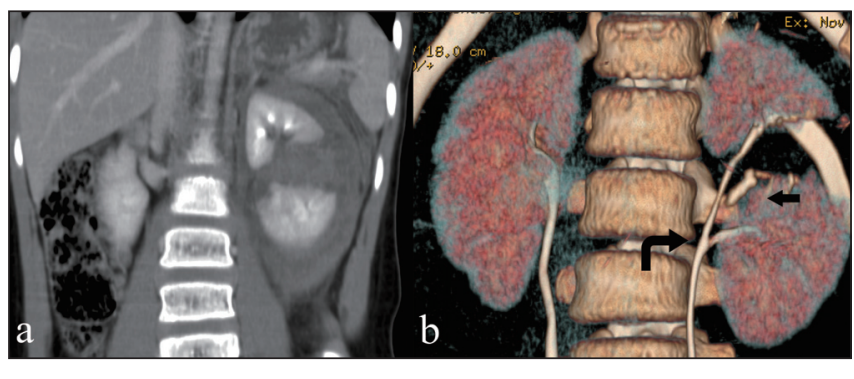

Figure 1: Contrast-enhanced computed tomography scan (a) shows a deep, full-thickness parenchymal fracture extending to the pelvis of the left kidney, with perirenal hematoma; scan with three-dimensional reconstruction (b) shows minimal urine extravasation (arrow) and upper left bifid ureter (curved arrow).

scan..$^{2-4}$ Internal stenting with or without percutaneous drainage is indicated if there is progressive urinoma or persistent urinary extravasation. Complete parenchymal fracture or significant urine extravasation may be less likely to resolve spontaneously; however, a CT scan with three-dimensional reconstruction may show a pre-existing anatomic abnormality, which may influence the decision for surgery. ${ }^{5}$

This article has been peer reviewed.

Competing interests: None declared.

Funding: This paper was supported by the Ta-Tung Kidney Foundation and the Mrs. Hsiu-Chin Lee Kidney Research Fund.

\section{REFERENCES}

1. Moore EE, Shackford SR, Pachter HL, et al. Organ injury scaling: spleen, liver, and kidney. J Trauma 1989;29:1664-6.

2. Henderson CG, Sedberry-Ross S, Pickard R, et al. Management of high grade renal trauma: 20-year experience at a pediatric level I trauma center. J Urol 2007; 178:246-50.

3. Salem HK, Morsi HA, Zakaria A. Management of high-grade renal injuries in children after blunt abdominal trauma. Experience of 40 cases. J Pediatr Urol 2007;3:223-9.

4. Rogers CG, Knight V, MacUra KJ, et al. High-grade renal injuries in children - Is conservative management possible? Urology 2004;64:574-9.

5. Michel LA, Lacrosse M, Decanniere L, et al. Blunt renal traumas: Contribution of spiral CT with three dimensional reconstruction to the surgical decision process? Int Surg 1996;81:377-81. 